\title{
Rutas de degradación del plaguicida n-metil carbamato carbaril
}

Routes of degradation of the pesticide carbaryl n-methylcarbamate

\section{JOSÉ CASTELLANOS ROZO}

Microbiólogo

MSc. Microbiología

Grupo de Investigación en Gestión Ambiental

Universidad de Boyacá, Colombia

joscastellanos@uniboyaca.edu.co

\section{JAQUELINE ARLETH GALVIS LÓPEZ}

Químico de Alimentos

Estudiante Maestría en Ciencias Biológicas Universidad Pedagógica y Tecnológica de Colombia

Grupo de Investigación en Gestión Ambiental

Universidad de Boyacá, Colombia

Jagalvis@uniboyaca.edu.co

Recibido: 09/12/2014

Aceptado: 26/02/2015 


\title{
RESUMEN
}

Carbaril (1-naftil $\mathrm{N}$-metilcarbamato) es un plaguicida perteneciente al grupo de los $\mathrm{N}$ - metilcarbamatos, se caracteriza por ser muy tóxico y moderadamente persistente en el medio ambiente, es utilizado extensivamente para el control de plagas en diferentes cultivos. Existen factores bióticos y abióticos que afectan el destino final de Carbaril en el ambiente. Se han reportado diferentes vías de degradación de este plaguicida, determinando que las bacterias son las principales degradadoras. El estudio de las vías de degradación es importante, debido a que muchos de los metabolitos podrían llegar a ser más tóxicos que el compuesto parental. La presente revisión busca establecer las rutas de degradación de Carbaril, los principales metabolitos y su impacto en el medio ambiente.

Palabras clave: N-metilcarbamatos, degradación, ecotoxicidad, xenobióticos, fotooxidación.

\begin{abstract}
Carbaryl (1-naphthyl-n-methylcarbamate) is a pesticide belonging to the group of $\mathrm{N}$ - methylcarbamate, $t$ is characterized by being very toxic and moderately persistent in the environment, it is extensively used for pest control in different crops. There are biotic and abiotic factors that affect the fate of Carbaryl in the environment. It has been reported different routes of degradation of this pesticide, determining that the bacteria are the main degradative. The study of degradation pathways is important, since many of the metabolites could be more toxic than the parent compound. This review seeks to establish routes of degradation of Carbaryl, the major metabolites and their impact on the environment.
\end{abstract}

Keywords: N - methylcarbamates, biodegradability, ecotoxicity, xenobiotics, photooxidation.

Castellanos, J. \& Galis, J. (2015). Rutas de degradación del plaguicida N-metilcarbamato 


\section{INTRODUCCIÓN}

Carbaril (1-naftil $\mathrm{N}$-metilcarbamato) es un plaguicida perteneciente al grupo de los $\mathrm{N}$ - metilcarbamatos, con fórmula molecular $\mathrm{C}_{12} \mathrm{H}_{11} \mathrm{NO}_{2}$, es un insecticida de amplio espectro cuya formulación comercial Sevin ${ }^{\circledR}$, es utilizado extensivamente para el control de plagas, en diferentes cultivos principalmente papa, cereales, legumbres, pasturas, árboles forestales. (IPCS, 1994; Agriculture and Agri-Food Canada, 1997; Rodríguez, 1996).

Se considera como un sólido cuyo peso molecular es de $201.20 \mathrm{~g} / \mathrm{mol}$, su punto de fusión es de $142^{\circ} \mathrm{C}$ y maneja una presión de vapor de $0.041 \mathrm{mPa}$ a $23.5^{\circ} \mathrm{C}$. Su solubilidad en agua es $120 \mathrm{mg} / \mathrm{L} \mathrm{a}$ $20^{\circ} \mathrm{C}$, es moderadamente soluble en disolventes orgánicos polares como la DMF ( $N, N$-dimetilformamida), el DMSO( dimetilsulfóxido) y la acetona, en otros disolventes carece de solubilidad como es el caso del hexano y del benceno. Fue sintetizado por primera vez por el doctor Lambrech en 1953 y lo introduce como plaguicida agrícola en el a ño de 1958 (Back, 1965; Kaufman, 1967). La EPA clasifica a Carbaril como un tóxico categoría II (Cranmer, 1986). Varios estudios demuestran que Carbaril se absorbe por inhalación, ingestión y vía tópica en mamíferos, aves, insectos y plantas. Se ha determinado que la dosis letal 50 oral aguda $\left(\mathrm{DL}_{50}\right)$ es de $108-840 \mathrm{mg} / \mathrm{Kg}$ para ratas, ratones, conejos, cobayas, perros, gatos y ciervos (Jorsaraei et al., 2014). Es altamente tóxico para invertebrados marinos y otras especies acuáticas, se han determinado $\mathrm{DL}_{50}$ para las especies de camarón de 5.7 $\mathrm{ppb}$, la concentración letal $\left(\mathrm{CL}_{50}\right)$ para Daphnia magna que va desde 1.7 hasta $26 \mathrm{ppb}$. También se ha determinado que la concentración letal $\left(\mathrm{CL}_{50}\right)$ aguda para los peces varía ampliamente, por ejemplo en escalas de 0.25 a 0.69 ppm para el salmón del Atlántico, la trucha de lago (Arcoiris) de 1.2 a 20 ppm (Koshlukova y Reed, 2014). Por otro lado, algunos reportes de aves registran toxicidad aguda que varía entre especies, la $\mathrm{DL}_{50}$ para el faisán (pato) es de $707 \mathrm{mg} / \mathrm{kg}$ mientras que para el pato ánade real es de $2500 \mathrm{mg} / \mathrm{kg}$ (Koshlukova y Reed, 2014). El carbaril es altamente tóxico para las abejas de miel con una $\mathrm{DL}_{50}$ aguda de $1 \mathrm{ppb}$, la lombriz de tierra (Lumbricus herculeus) es particularmente susceptible con un valor $\mathrm{CL}_{50}$ en suelo de 0.5 ppm en períodos cortos (5 horas) (Eisler, 1985; Lima et al., 2011).

Carbaril en el ser humano inhibe la acetilcolinesterasa, puede provocar disfunción del sistema reproductivo y endocrino e influir en la proliferación celular de procesos cancerosos, sin embargo no se considera mutagénico (Moreno, 2002).

El efecto del Carbaril en el medio ambiente podría ser mitigado si se conoce el efecto que tienen diferentes factores abióticos, como la temperatura, $\mathrm{pH}$, contendido de materia orgánica, adición de nutrientes, y factores bióticos como el metabolismo realizado por animales, plantas y microorganismos (Megharaj et al., 2011). 
La degradación de Carbaril de manera biótica y abiótica conlleva a rutas degradativas que generan metabolitos, los cuales podrían llegar a ser más tóxicos que el compuesto parental, por lo tanto, la presente revisión pretende estudiar los principales factores que afectan la degradación del Carbaril, hace énfasis en el destino ambiental del plaguicida, sus transformaciones parciales, productos metabólicos y el efecto que ejercen sobre el medio ambiente.

\section{DEGRADACIÓN DE CARBARIL EN EL AMBIENTE}

La degradación de Carbaril se ve afectada por factores extrínsecos como la temperatura, pH, anoxia y alto contenido de materia orgánica (Hanazato y Yasuno, 1989; Lartigues y Garrigues, 1995). En aguas y suelos cuyo pH es básico, la degradación de Carbaril es rápida comparada con la degradación a pH neutro y ácido. Se ha reportado que la degradación de Carbaril en condiciones alcalinas (pH 8.0) es de 1.8 días (Aly y El-Dib; 1971) mientras que a pH neutro (7.0-7.4), la vida media de este plaguicida es de 10.5 días (Howard, 1991), a pH ácido (5.0) Larkin y Day, 1986, demostraron que la vida media de la degradación de Carbaril en el agua, podría extenderse hasta cuatro años.

En otros estudios se ha concluido que en aguas y suelos con pH alcalino y temperatura de $20^{\circ} \mathrm{C}$, el Carbaril se hidroliza rápidamente a 1-naftol, metilamina y dióxido de carbono (Stewart et al., 1967; Moreno, 2002; Durkin y King, 2008; Venkateswarlu et al., 1980; Rajagopal et al., 1983; Gunasekara et al., 2008; Kazano et al., 1972).

También se ha determinado que la degradación del Carbaril en el agua y en el suelo, podría darse por la acción de la luz (foto degradación) (Larkin y Day, 1986; Bollag y Liu, 1971; Rajagopal et al., 1984; Ramanand et al., 1988; Sudy et al., 1972). El mayor producto de la fotodegradación es el metabolito 1-naftol, que luego es fotooxidado a 2-hidroxi-1,4-nafto-quinona en medio básico (Montgomery, 2007).

La degradación de Carbaril también se ve influenciada por el contenido de materia orgánica, lo cual hace que el plaguicida persista o no en el medio ambiente. Estudios realizados por Johnson y Stansbury en el año 1965, encontraron que en un suelo franco arenoso, la vida media de este compuesto era de ocho días (Kazano et al., 1972), no obstante Ahmad y colaboradores en el 2004, investigaron la biodisponibilidad y degradación de Carbaril en un suelo con alto contenido de materia orgánica con una larga historia de aplicación, sus resultados demostraron que el Carbaril persiste en el suelo. 


\section{RUTAS DE DEGRADACIÓN DE CARBARIL EN PLANTAS Y ANIMALES}

La degradación de Carbaril es llevada a cabo en el medio ambiente, por factores bióticos como el metabolismo de animales, plantas, microorganismos, tanto en animales como en plantas es muy similar, su metabolización se da por hidrólisis e hidroxilación del anillo, generando con ello numerosos metabolitos como son 1-naftol, 5,6-dihidro-5,6-hidroxicarbaril, 1-naftil-N-hidroximetil-carbaril, 4-hidroxiCarbaril, 5-hidroxicarbaril (Kuhr y Casida, 1967; Kuhr, 1970; Lima et al., 2011), estos metabolitos a su vez sufren conjugaciones que dan lugar a sulfatos, lucorónidos y compuestos mercapturados, que llegan a ser solubles y que son eliminados en la orina (CASAFE,1995). (Figura 1). Se ha determinado que en los animales, los niveles más altos de Carbaril y su principal metabolito 1-naftol, se encuentran en el riñón, sangre, hígado y cerebro (Koshlukova y Reed, 2014).

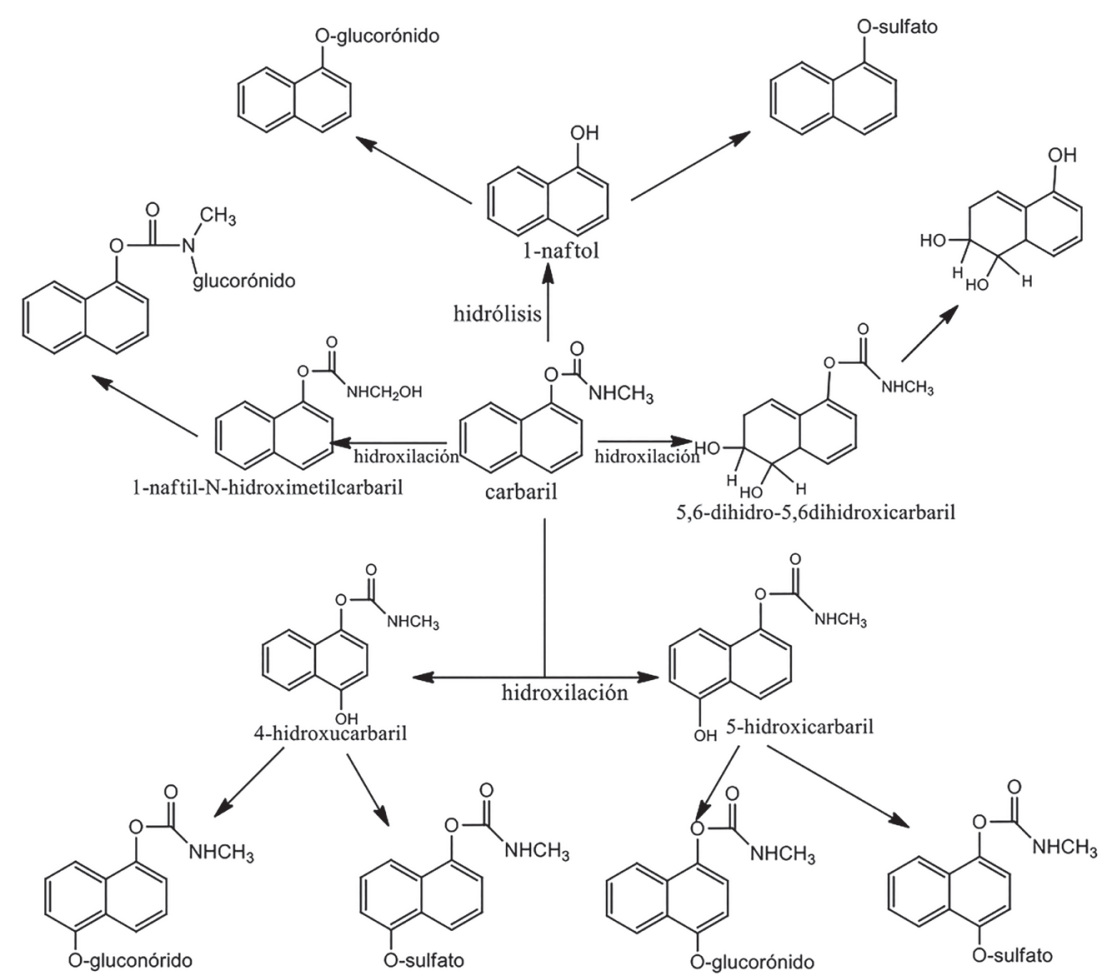

Figura 1. Metabolitos primarios de Carbaril producidos por el metabolismo de animales y plantas. IPCS (International Programme on Chemical Safety). 1994. 


\section{RUTAS DE DEGRADACIÓN DE CARBARIL POR BACTERIAS}

Estudios realizados por Parkin y Shelton (1994), concluyeron que las bacterias son las principales responsables de la degradación de carbaril. Ensayos hechos por Parekh y colaboradores en 1994, determinaron que cuando los suelos son tratados con cloranfenicol, se reduce la tasa de degradación de carbofuran y Carbaril con respecto a suelos no tratados, lo cual indica que las bacterias del suelo son las principales responsables de la degradación de estos plaguicidas.

Desaint y colaboradores en el 2000, concluyeron con sus investigaciones, que el uso extenso de estos compuestos en suelos agrícolas, ha inducido mecanismos de adaptación genética en los microorganismos, conllevando a la síntesis de enzimas que oxidan, hidrolizan e hidroxilan Carbaril, permitiendo su utilización como única fuente de carbono y nitrógeno.

Se han reportado diferentes bacterias que degradan Carbaril como única fuente de carbono y/o nitrógeno para su crecimiento como Achromobacter sp. (Bollag y Liu, 1971; Sudy et al., 1972; Karns y Tomasek, 1991), Rhodococcus sp. 12038 (Larkin y Day, 1986), Bacillus sp. (Rajagopal et al., 1984), Pseudomonas sp., 50552 y 50581 (Chapalamadugu y Chaudhry, 1991), Pseudomonas sp., 12043 (Larkin y Day, 1986), Blastobacter sp., M501 (Hayatsu y Nagata, 1993), Arthrobacter sp., RC100 (Hayatsu et al., 1999), Rhizobium sp., (Hashimoto e t a l., 2002 ), Aminobacter ciceronei nov. ER2 (Topp et al., 1993), Sphingomonas sp., CF06 (Ogram et al., 2000), Micrococcus sp., (Doddamani y Ninnekar 2000).

La hidrólisis enzimática del enlace metilcarbamato es el mecanismo primario de inactivación del Carbaril en el ambiente. La hidrólisis del enlace carbamato podría ocurrir por cualquiera de las dos vías, por rompimiento del enlace éster (el grupo carbonilo del ácido $\mathrm{N}$-metilcarbámico unido al fenol) o por rompimiento del enlace amida, tanto con la amidasa o con la esterasa, el producto de la hidrólisis es idéntico, dada la inestabilidad del enlace del ácido N-metilcarbamato produciendo 1-naftol, metilamina y dióxido de carbono (Castellanos, R. J., \& Rache L. 2013). Entre los microorganismos aislados y caracterizados a nivel mundial que hidrolizan Carbaril se encuentra: Sphingomonas sp CF06 (Ogram et al., 2000), Sphingobium qiguonii sp. Nov (Yan et al., 2010), Aminobacter ciceronei nov. ER2 (Topp et al., 1993), Rhizobium sp., (Hashimoto et al., 2002 ), Blastobacter sp., M501 (Hayatsu y Nagata, 1993) (Figura 2a).

Sin embargo, muchas de las bacterias que hidrolizan carbaril, son incapaces de degradar el metabolito denominado 1-naftol. Inclusive, se ha comprobado que el metabolito 1-naftol, es más tóxico que el compuesto parental carbaril, generando un daño más grave para el medio ambiente (Chapalamadugu y Chaudhry ,1991). 
Estudios realizados por Sato y colaboradores (1999), reportaron dos aislamientos bacterianos que degradan rápidamente Carbaril, estos aislamientos fueron identificados como Arthrobacter sp., basados en el contenido de guaninas y citosinas, la composición de ácidos grasos, la presencia de menaquinona isopregnenolona y el análisis del gen $16 \mathrm{~S}$ rRNA. La cepa Arthrobacter sp., 19B, degradó completamente $50 \mathrm{mg} / \mathrm{L} /$ día de Carbaril en medio mínimo de sales minerales. Análisis por cromatografía de gases y espectrometría de masas evidenciaron que el principal producto de la degradación de Carbaril por Arthrobacter sp., es el metabolito 1-naftol, el cual se transforma en los metabolitos secundarios: 1,4-naftalenodiona y 1,4-naftalenodiol, lo que sugiere una misma vía de degradación del anillo aromático de Carbaril (Figura 2b).

Swetha y Phale (2005), estudiaron tres cepas de Pseudomonas sp. C4, C5, y C6 que utilizaron Carbaril como única fuente de carbono, el objetivo fue determinar la ruta catabólica, para ello las cepas aisladas del suelo contaminado con Carbaril, se cultivaron en un medio mínimo de sales a $25^{\circ} \mathrm{C}$ y a $200 \mathrm{rpm}$, el crecimiento bacteriano fue medido por espectrofotometría a $540 \mathrm{~nm}$. Se aislaron e identificaron los metabolitos por la técnica de cromatografía de gases acoplada a espectrofotometría de masas (GC/MS). Los resultados obtenidos determinaron que la ruta de degradación era 1-naftol con la subsiguiente conversión a 1,2-dihidroxi- naftaleno, 2-hidroxibenzal ácido pirúvico, ácido gentísico, el cual finalmente entra al ciclo de Krebs y se convierte en dióxido de carbono y agua (Figura 2c).

En trabajos en los que se estudiaron los aislamientos de Pseudomonas sp. 12043 y Rhodococcus sp. 12038, se demostró que las bacterias crecían en presencia de Carbaril utilizándolo como única fuente de carbono y nitrógeno, aunque las tasas de degradación de carbaril fueron lentas, ambos aislamientos utilizaron 1-naftol como única fuente de carbono produciendo salicilaldehído, ácido salicílico y ácido gentísico (Larkin y Day, 1986).

Estudios realizados por Doddamani y Ninnekar en el 2001, aislaron e identificaron una especie de Micrococcus sp., a partir de suelos contaminados con Carbaril por la técnica de cultivo enriquecido en medio mineral Seubert, con Carbaril $(0.2 \% \mathrm{~g} / \mathrm{L}$, pH de 6.8) como única fuente de carbono. Micrococcus sp., hidrolizó Carbaril generando 1-naftol, metilamina y dióxido de carbono, con la subsiguiente metabolización del 1-naftol vía Gentisato. Además de degradar carbaril pudo utilizar otros compuestos aromáticos como sustratos de crecimiento, entre ellos carbofuran, naftaleno y 1-naftol como única fuente de carbono (Figura 2d). 


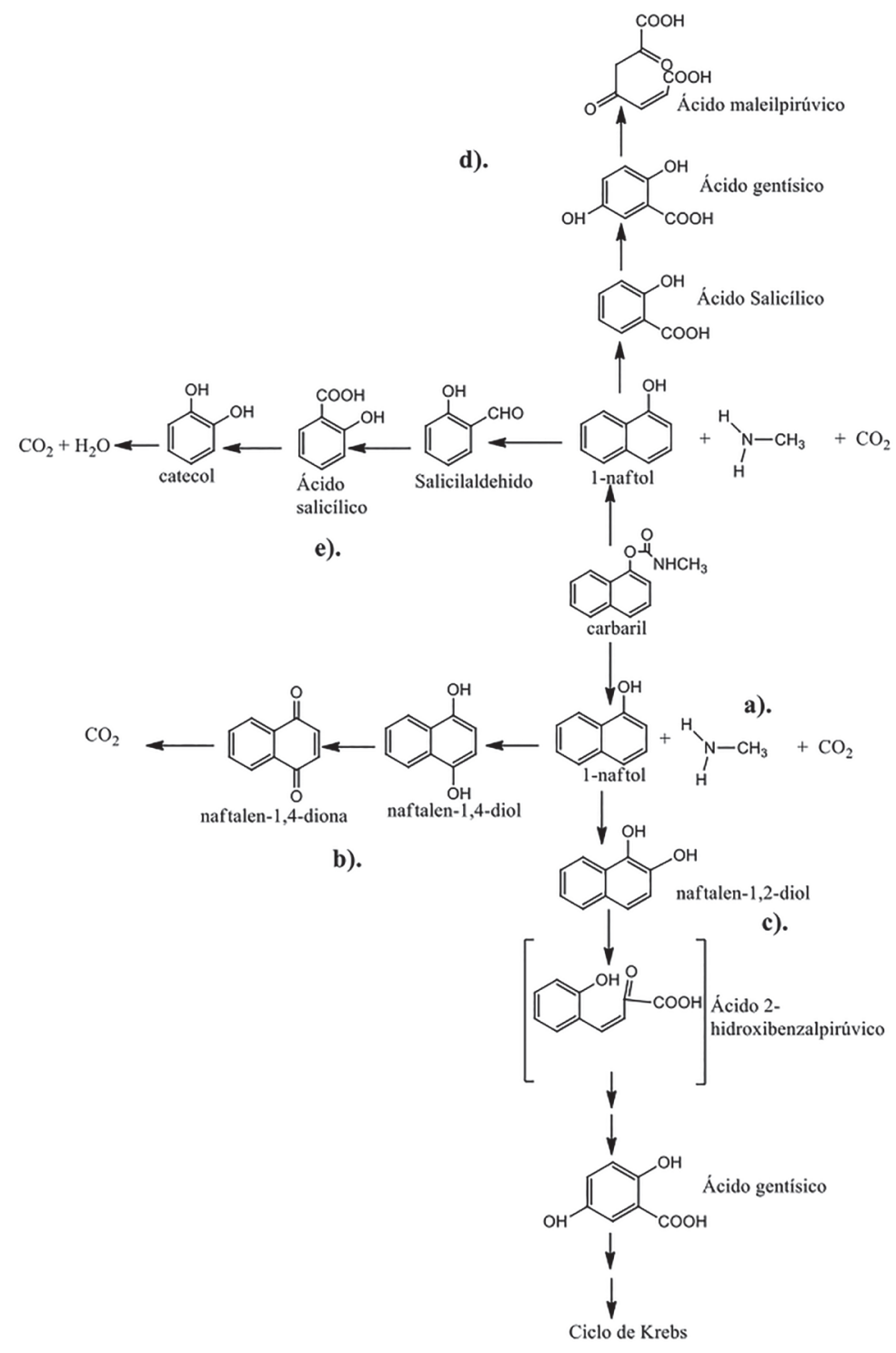

Figura 2. Rutas empleadas por los microorganismos para la degradación de Carbaril. a). Ruta propuesta por Topp et al., 1993; McDonald et al., 2005 para Aminobacter ciceronei nov. ER2 b). Ruta propuesta por Sato et al., 1999 para Arthrobacter spp. c). Ruta propuesta por Swetha et al., 2007 para Pseudomonas sp. C4 d). Ruta propuesta por Doddamani y Ninnekar (2000) para Micrococus sp. e). Ruta propuesta por Chapalamadugu y Chaudhry (1990) para el consorcio microbiano de Pseudomonas sp.

Fuente: Modificado por los autores. 
Naqvi y colaboradores (2011), evaluaron la degradación de Carbaril en suelo por adición de nitrato de sodio al $1 \%$ y dihidrogenofosfato de potasio al $0,1 \%$ y por adición de un consorcio bacteriano, el cual estaba constituído por Micrococus arborescens, Pseudomonas aeruginosa, Brachybacterium sp., y Salsuginibacillus Kocurii. Los resultados demostraron un aumento significativo en la degradación de Carbaril al transcurrir el tiempo, registrando niveles de degradación hasta del 85\% en 21 días. No obstante se ha determinado en estudios con 2-clorobenzoato que la adición de nutrientes, podría reducir la degradación (Hernández et al., 1991).

Otros estudios realizados por Chapalamadugu y Chaudhry (1991), aislaron dos Pseudomonas sp. El aislamiento Pseudomonas sp. 50581 hidrolizó carbaril produciendo 1-naftol, metilamina y dióxido de carbono, sin la subsiguiente degradación de 1-naftol. Pseudomonas sp. 50552 no pudo degradar Carbaril, pero pudo mineralizar el 1-naftol. Por lo tanto establecieron un consorcio microbiano de Pseudomonas sp., que permitió la rápida degradación de Carbaril produciendo salicilaldehído, ácido salicílico, catecol, hasta dióxido de carbono y agua (Figura 2e).

\section{CONCLUSIONES Y RECOMENDACIONES}

Carbaril se hidroliza en agua y suelo de manera abiótica y biótica produciendo como principal metabolito 1-naftol, sin embargo el 1-naftol es más tóxico que el compuesto parental. Carbaril puede ser metabolizado por animales, plantas y microorganismos utilizando diferentes rutas de degradación como la hidrólisis e hidroxilación del anillo bencénico. Sin embargo las bacterias tienen un efecto significativo en la degradación. Se ha comprobado que existe una gran diversidad de microorganismos degradadores del plaguicida Carbaril. No obstante, son pocas las bacterias que tienen la capacidad de degradar 1-naftol como única fuente de carbono hasta dióxido de carbono y agua. La degradación de Carbaril en el ambiente hasta dióxido de carbono y agua, podría ser acelerada si se crean consorcios de microorganismos a los cuales se les fortifique con fosfatos y nitratos como sustratos para su crecimiento.

\section{AGRADECIMIENTOS}

Los autores agradecen a la Universidad de Boyacá por el apoyo otorgado en el financiamiento del proyecto Macro denominado: Degradación de los plaguicidas N-metilcarbamatos Carbofuran y Carbaril por la cepa bacteriana Sphingobium sp, en condiciones in vitro. 


\section{REFERENCIAS BIBLIOGRÁFICAS}

Ahmad, R., R.S. Kookana, M. Megharaj and A.M. Alston. (2004). Aging reduces the bioavailability of even a weakly sorbed pesticide (carbaryl) in soil. Environ. Toxicol. Chem., 23(9): 2084-9.

Agriculture and Agri-food Canada. (1997). Agriculture in harmony with nature: Strategy for eviromentally sustainable Agriculture and Agri-Food Canada development in Canada. http://www.agr.ca/ policy/envharmon/docs/strate.pdf. Consultado el 15 Febrero 2015.

Aly, D.M \& El-Dib M.A. (1971). Studies on the persistence of some carbamate insecticides in the aquatic environment. Hydrolysis of sevin, baygon, pyrolan and dimetilan in water. Water Res. 5(12): 1191-1205.

Back, R.C. (1965).Significant developments in eight years with Sevin insecticide, J. Agr. Food Chem. 13(3): 198-199.

Bonner, M.R., Lee W.J \& Sandler D. (2005). Occupational exposure to Carbofuran and the incidence of cancer in the agricultural health study. Environ. Health. Perspect. 113(3):285-289.

Bollag, J.M \& Liu, S.Y. (1971). Degradation of sevin by soil microorganisms. Soil Biol. Biochem. 3(3): 337-345.

Cranmer, M.F. (1986). Carbaryl a tocicological review and risk analysis. Neurotoxicology 7(1): 247-328.

CASAFE (Cámara de Sanidad Agropecuaria y Fertilizantes de la República Argentina). (1995). Guía de Productos Fitosanitarios para la República Argentina, Buenos Aires. 891 p.

Castellanos, R. J., \& Rache L. (2013). Microorganismos, enzimas, plásmidos y genes involucrados en la degradación de plaguicidas n-metilcarbamatos. Rev Int. Contam. Ambie. 29 (Número especial sobre plaguicidas) 105-119.

Chapalmadugu, S. \& Chaudhry, G.R. (1991). Hydrolysis of Carbaryl by Pseudomonas sp. and construction of a microbial consortium that completely metabolizes Carbaryl. Appl. Environ. Microbiol. 57(1): 744-750.

Desaint S.A., Hartmann, N., Parekh, H. \& Fournier, J. C. (2000). Genetic diversity of carbofuran-degrading soil bacteria. FEMS Microbiol Ecol. 34 (2): 173 - 180. 
Durkin, P.R \& King, C. (2008). Carbaryl human health and ecological risk assessment revised final report. Syracuse environmental research associates, Inc. USDA forest service contract. http://www. fs.fed.us/foresthealth/pesticide/pdfs/052-01-05aCarbaryl.pdf. Consultado el 20 febrero 2015.

Eisler, R. (1985). Carbofuran Hazards to Fish, Wildlife, and Invertebrates: A Synoptic Review. U.S. Fish Wildl. Serv. Biol. Rep. 85(13): 13-36.

Gunasekara, A.S., Alrubin, K.S., Goh, F.C. Spurlock, J \& Tjeerdema, R.S. (2008). Environmental fate and toxicology of carbaryl. En: M.Whitacre (ed.) Rev.Environ. Contam. Toxicol. 196. 12(2): 145-248.

Hanazato, T. \& Yasuno, M. (1989). Effects of Carbaryl on the spring zooplankton communities in ponds. Environ. Pollut. 56(1):1-10.

Hayatsu, M. \& Nagata, T. (1993). Purication and characterization of carbaryl hydrolase from Blastobacter sp. M501. Appl. Environ. Microbiol. 59 (1): 2121-2125.

Hayatsu, M., Hirano, M. \& Nagata, T. (1999). Involvement of two plasmids in the degradation of carbaryl by Arthrobacter sp. Strain RC100. Appl. Environ. Microbiol. 65 (3):1015-1019.

Hashimoto, M., Fukui. M., Hayano, K., Hayatsu, M. (2002). Nucleotide sequence and genetic structure of a novel carbaryl hydrolase gene (cehA) from Rhizobium sp. Strain AC100. Appl. Environ. Microbiol. 68 (3):1220-1227.

Hernández, B.S., F.K. Higson, R. Kondrat and D.D. Focht. (1991). Metabolism of and inhibition bychlorobenzoates in Pseudomonas putida P111. Appl. Environ. Microbiol., 57: 3361-3366.

Howard, P.H. (1991). Handbook of environmental fate and exposure data for organic chemicals. Pesticides. Lewis Publishers Inc., Chelsea, and MI. En: Kamrin, M.A.Pesticides profiles. Toxicity, Environmental Impact, and Fate. Lewis Publishers. En http://www.finagro.com.co/html/i_portals/index.php consultado el 22 de Enero del 2015.

INS (Instituto Nacional de Salud. 2012). Programa de Vigilancia Epidemiológica de Plaguicidas Organofosforados y Carbamatos. 18 (20): 18-72

IPCS (International Programme on Chemical Safety). (1994). Environmental Health Criteria 153.Carbaryl.WorldHealth.Organization.Geneva. http:/www.inchem.org/documents/ehc/ehc/ehc153.htm. Consulta el 21 de enero de 2015. 
Johnson, D.P \& Stansbury, H.A. (1965). Adaptation of sevin insecticide (carbaril) residue method to various crops. J. Agric. Food Chem. 13 (3): 235-238.

Jorsaraei, A., Maliji, G., Azadmehr, A., Moghadamnia, A \& Faraji, A. (2014). Immunotoxicity effects of carbaryl in vivo and in vitro. Environ.Toxicol. Chem. 38(3):838-844.

Karns, J.S \& Tomasek, P.H. (1991). Carbofuran hydrolase purification and properties. J. Agric. Food Chem. 39(1): 1004-1008.

Kaufman, D.D. (1967). Degradation of Carbamete herbicides in Soil. J. Agr. Food Chem. 15(4): 582-591.

Kazano, H.P., Kearney, C \& Kaufman, D.D. (1972). Metabolism of methylcarbamate Insecticides in soil. J. Agr. Food Chem. 2(1): 975-978.

Koshlukova, S.E \& Reed, N.R. (2014). Carbaryl. Encyclopedia of Toxicology. Elsevier. 668-672.

Kuhr, R.J. (1970). Metabolism of carbamate insecticide chemicals in plants and insects.J.Agr.Food Chem.18:1023-1030.

Kuhr, R.J. \& Casida, J. (1967). Persistent glycosides of metabolites of methyl carbamate insecticide chemical formed by hydroxylation in bean plants. J.Agr.Food chem.15:814-828.

Ramanand, K \& Panda, S. (1988).Mineralization of Carbofuran by a Soil Bacterium. Appl. Environ and Microbiol. 54(1):2129-2133.

Larkin, M.J \& Day, M.J. (1986). The metabolism of Carbaryl by three bacterial isolates, Pseudomonas sp. (NCIB 12042 and 12043) and Rhodococcus sp. (NCIB 12038) from Garden Soil. J. Appl. Bacteriology. 60(1): 233-242.

Lartigues, S.B \& Garrigues, P.P. (1995).Degradation kinetics of organophosphorus and organonitrogen pesticides indifferent waters under various environmental conditions. Environ. Sci. Technol. 29(5): 1246-1254.

Lima, M Soares, Amadeu M.V.M \& Loureiro, S. (2011). Combined effects of soil moisture and carbaryl to earthworms and plants: Simulation of flood and drought scenarios. Environ. Pollut. 159 (7): 1844-1851. 
Megharaj M., Ramakrishnan B., Venkateswarlu K., Sethunathan N. y Naidu R.( 2011). Bioremediation approaches for organic pollutants: A critical perspective. Environ. Int. 37, 1362-1375.

Montgomery, J. (2007). Groundwater Chemicals Desk Reference, Fourth Edition pág. 200.

Moreno, M. (2002). Desarrollo de un inmunoensayo multianalito, basado en anticuerpos monoclonales, para la determinación de plaguicidas $\mathrm{N}$-metilcarbamatos en frutas y hortalizas.Tesis para optar al Grado de Doctor en Ciencias Químicas. Universidad Politécnica de Valencia. 131p.

Naqvi, T.A., Kanhar, N.A., Shar, A.H., Hussain, M \& Ahmed, S. (2011). Microcosm studies for the biodegradation of Carbaryl in soil. Pakistán J. Bot. 43(2): 1079-1084.

Ogram, A.V., Duan, Y.P., Trabue, S.L., Feng, X., Castro, H \& Ou, L.T. (2000). Carbofuran degradation mediated by three related plasmid systems. FEMS Microbiol. Ecol. 32(3): 197-203.

Parkin, T.B \& Shelton D.R. (1994). Modeling Environmental Effects on Enhanced Carbofuran Degradation. Pesticide Science. 40(2):163-168

Rajagopal, B.S., Chendrayan, K., Reddy, B.R \& Sethunathen, N. (1983). Persistence of Carbaryl in flooded soils and its degradation by soil enrichment cultures. Plant Soil. 73(1): 35-45.

Rajagopal, B.S., Brahmaprakash, G.P., Reddy, B.R., Singh, U.D \& Sethunathan, N. (1984). Effects and persistence of selected carbamate pesticides in soil. Residue Rev. 93(1): 1-199.

Rodríguez, A. (1996). Consideraciones al manejo de plagas y enfermedades de la papa en Colombia. En: Papas Colombianas con el Mejor Entorno Ambiental, Editorial Comunicaciones y Asociados-Fedepapa. 122-126pp.

Sato, H., Takeuchi, T \& Sakai, K.L. (1999). Temporal cortex activation during speech recognition an optical topography study cognition. 73(2): 55-66.

Stewart, N.E., Millemann, R.E \& Breese, W.P. (1967). Acute toxicity of the insecticide Sevin and its hydrolytic product 1-naphthol to some marine organisms. Trans. Am. Fish. Soc. 96 (3):25-116.

Sudy, R.K., Sud, A.K \& Gupta, G. (1972). Degradation of Sevin (1-Napthyl-N-methyl carbamate) by Achromobacter sp. Arch.Microbiol. 87(1):353-358.

Swetha, V. \& Phale, P.S.( 2005). Metabolism of Carbaryl via 1,2-Dihydroxynaphthalene by Soil Isolates Pseudomonas sp. Strains C4, C5, and C6. Appl. Environ. Microbiol. 71(10): 5951-5956. 
Taylor \&Francis group, LLC. EE.UU.

Topp, E., Hanson, R.S., Ringelberg, D.B., White, D.C \& Wheatcroft, R. (1993). Isolation and characterization of an $\mathrm{N}$-methylcarbamate insecticide-degrading methylotrophic bacterium. Appl. Environ. Microbiol.59 (10):3339-3349.

Venkateswarlu, K., Chendrayan, K \& Sethunathan, N. (1980). Persistence and biodegradation of carbaryl in soils. J. Environ. Sci. Health, 15B: 421-429.

Yan, Q-X; Wang, Y-X; Li, S-P; Li, W-J \& Hong Q. (2010). Sphingobium qiguonii sp. nov., a carbaryl degrading bacterium isolated from a wastewater treatment system International. Int. J.

Syst Evol Microbiol. 60: 2724-2728. 